\title{
ENVELHECIMENTO ACELERADO EM SEMENTES DE CUBIU (Solanum sessiliflorum Dunal) ${ }^{1}$
}

\author{
Márcio Dias Pereira², Sebastião Martins Filho ${ }^{3}$
}

\begin{abstract}
ACCELERATED AGING

OF CUBIU (Solanum sessiliflorum Dunal) SEEDS

Cubiu is a native plant of the Amazon that has attracted attention due to its nutritional and pharmaceutical properties. The objective of this study was to adequate the methodology of accelerated aging test for vigor evaluation of Solanum sessiliflorum seeds. Four seed lots of Solanum sessiliflorum were tested for germination, first counting, plantule length, emergence and germination speed index. For accelerated aging, the temperatures $41^{\circ} \mathrm{C}, 42^{\circ} \mathrm{C}$, and $45^{\circ} \mathrm{C}$ were tested for 24,48 , 72 , and 96 hours. The accelerated aging test was efficient for vigor evaluation of Solanum sessiliflorum seeds, with results similar to those found for first germination counting, plantule length, emergence and germination speed index, especially when conducted at $41^{\circ} \mathrm{C}$, for 72 or 96 hours.
\end{abstract}

KEY-WORDS: Solanum sessiliflorum Dunal; physiological quality; vigor; deterioration.

\section{INTRODUÇÃO}

O cubiu, também chamado de maná, topiro e tomate de índio, é uma solanácea originária da Amazônia Ocidental, domesticada por povos antigos. É encontrada na Amazônia brasileira, peruana e colombiana. A planta é um arbusto ereto e ramificado, que cresce de $1 \mathrm{~m}$ a $2 \mathrm{~m}$ de altura, e é cultivada em diversos tipos de solos da Amazônia, zona da mata pernambucana e em algumas localidades da região Sudeste (Silva Filho 1996). Produz frutos comestíveis, com conteúdo considerável de nutrientes, que podem ser consumidos in natura, nas formas de sucos, doces e geleia. É usado como tempero de pratos à base de peixe, carne e frango e amplamente empregado na medicina tradicional, para controlar altos níveis de colesterol, ácido úrico e açúcar no sangue (Silva et

\section{RESUMO}

O cubiu é uma planta nativa da Amazônia, que tem despertado interesse pelas suas características nutricionais e farmacêuticas. O presente trabalho teve por objetivo adequar a metodologia do teste de envelhecimento acelerado, para a avaliação do vigor de sementes de cubiu. Para isto, sementes de quatro lotes foram submetidas a testes de germinação, primeira contagem, comprimento de plântula, emergência e índice de velocidade de germinação (IVG). Para o teste de envelhecimento acelerado, foram testadas as temperaturas de $41^{\circ} \mathrm{C}, 42^{\circ} \mathrm{C}$ e $45^{\circ} \mathrm{C}$, durante os períodos de $24,48,72$ e 96 horas. O teste de envelhecimento acelerado foi eficiente para avaliação do vigor das sementes de cubiu, fornecendo informações semelhantes às obtidas na primeira contagem de germinação, comprimento de plântula, emergência e IVG, principalmente quando conduzido a $41^{\circ} \mathrm{C}$, por 72 ou 96 horas.

PALAVRAS-CHAVE: Solanum sessiliflorum Dunal; qualidade fisiológica; vigor; deterioração.

al. 1995). Fora da Amazônia, o fruto ganhou destaque e já é utilizado para a produção de sucos, além de ter se tornado alvo do interesse de pesquisadores, em várias instituições brasileiras que têm estudado suas características agronômicas e tecnológicas.

A propagação da espécie é seminal e o seu manejo, desde a semeadura até o plantio definitivo, pode ser semelhante ao utilizado no cultivo de outras solanáceas, como o tomateiro, pimentão, jiló ou berinjela. A produção de frutos inicia-se seis meses após o transplante, com produção econômica de frutos durante três meses (Silva Filho 1998).

O formato dos frutos varia de acordo com o genótipo, podendo ser redondo, achatado, quinado, cilíndrico ou cordiforme. A coloração varia entre verde, quando imaturo; amarelo, quando maduro; e marrom-avermelhado, no estádio mais avançado de

1. Trabalho recebido em fev./2009 e aceito para publicação em ago./2010 (nº registro: PAT 5608/ DOI: 10.5216/pat.v40i3.5608).

2. Universidade Federal de Viçosa, Centro de Ciências Agrárias, Departamento de Fitotecnia, Viçosa, MG, Brasil.

E-mail: marcio.pereira@ufv.br.

3. Universidade Federal de Viçosa, Centro de Ciências Exatas, Departamento de Estatística, Viçosa, MG, Brasil.

E-mail: martinsfilho@ufv.br. 
maturidade. Os frutos cilíndricos têm, em geral, 4 lóculos e os cordiformes, redondos e achatados de 6 a 8 , podendo haver variações no número de lóculos, nos frutos de uma mesma planta. O fruto é, geralmente, coberto de pêlos curtos e quebradiços, que são facilmente removidos, esfregando-os com as mãos (Silva Filho 1998). Cada fruto, cujo peso pode variar de $20 \mathrm{~g}$ a 450 g, contém de 500 a 2.000 sementes glabras, ovaladas e achatadas ( 1.000 sementes pesam $1 \mathrm{~g}$ ), com coloração amarela, comprimento de $3,2 \mathrm{~mm}$ a $4,0 \mathrm{~mm}$, testa lisa, endosperma duro, cotilédones ovóides, inteiros, peninérveos e longo peciolados. A geminação é epígea e fanerocotiledonar (Silva Filho 2002).

Apesar da grande utilidade dos frutos de cubiu e do crescente interesse da pesquisa, ainda são poucos os estudos publicados sobre a espécie, principalmente no tocante à tecnologia de produção de sementes. Para os programas de qualidade internos, das empresas produtoras de sementes, é importantíssimo conhecer as metodologias que proporcionem respostas sobre as características de vigor das sementes e não só a detenção da metodologia do teste de germinação. A necessidade das empresas de se avaliar o vigor de sementes, para seu controle interno de qualidade, exige que se ajustem as metodologias dos testes de vigor para cada cultura (Dias \& Marcos Filho 1996).

Muitos são os testes que avaliam a qualidade fisiológica das sementes em condições de estresse, simulando as adversidades sofridas pelas sementes. Dentre eles, tem sido cada vez mais usado o de envelhecimento acelerado, por apresentar resultados confiáveis, uma vez que avalia o comportamento das sementes quando submetidas a condições de alta temperatura e alta umidade relativa do ambiente, procurando-se, assim, estimar o potencial relativo de armazenamento dos lotes (Delouche \& Baskin 1973). O teste de envelhecimento acelerado tem como base o fato de que a taxa de deterioração das sementes aumenta, consideravelmente, pela sua exposição à temperatura e umidade relativa elevadas, sendo estes os fatores ambientais mais relacionados à deterioração das sementes. Desse modo, lotes de sementes com alto vigor manterão sua viabilidade, após serem submetidos ao estresse, enquanto os de baixo vigor terão sua viabilidade reduzida (AOSA 1983).

Muitos trabalhos têm sido desenvolvidos, empregando-se o teste em sementes de diversas oleráceas, como a cebola (Piana et al. 1995), o quiabo (Torres \& Carvalho 1998), a cenoura (Spinola et al.
1998, Ribeiro 2000, Rodo et al. 2000), o pimentão (Panobianco \& Marcos Filho 1998), o tomate (Rodo et al. 1998, Panobianco \& Marcos Filho 2001), o pepino (Bhering et al. 2000), a melancia (Bhering et al. 2003), a rúcula (Ramos et al. 2004) e a alface (Ribeiro 2000, Franzin et al. 2004). Porém, não se encontram informações sobre a aplicação deste teste para a avaliação do vigor de sementes de cubiu.

De acordo com Marcos Filho (1999), a pesquisa deve fornecer informações para sanar dúvidas como, por exemplo, as combinações de temperatura e tempo de exposição, para as diferentes espécies. Em sementes menores, como as de cubiu, com tamanho e forma muito semelhantes às do tomate, $o$ teste de envelhecimento acelerado pode apresentar limitações. Sementes pequenas absorvem água mais rapidamente e de maneira desuniforme, durante o envelhecimento, o que pode acelerar o processo de deterioração ou resultar em comportamento variável entre as sementes de uma amostra, interferindo na precisão dos resultados. Diante da importância do teste de envelhecimento acelerado, para se avaliar a qualidade fisiológica de lotes de sementes, e da escassez de informações sobre a metodologia adequada para as sementes de cubiu, o objetivo deste trabalho foi adaptar a metodologia do teste de envelhecimento acelerado para sementes de cubiu.

\section{MATERIAL E MÉTODOS}

Os ensaios foram desenvolvidos no Laboratório de Análises de Sementes Florestais do Departamento de Engenharia Florestal da Universidade Federal de Viçosa (MG), utilizando-se quatro lotes diferentes de sementes de cubiu, variedade Santa Luzia, provenientes do Instituto de Pesquisas da Amazônia.

Foram realizados os seguintes testes e determinações: 1) Teor de água: realizado pelo método da estufa, a $105 \pm 3^{\circ} \mathrm{C}$, por 24 horas, utilizando-se duas subamostras para cada lote. Este procedimento foi efetuado antes e após os períodos de envelhecimento, sendo os resultados expressos em percentagem na base úmida (Brasil 2009); 2) Germinação: conduzido com quatro repetições de 50 sementes, distribuídas sobre duas folhas de papel germitest, em caixas plásticas transparentes $(11,0 \mathrm{~cm} \times 11,0 \mathrm{~cm} \times 3,5 \mathrm{~cm})$, tipo gerbox, umedecidas com água destilada, em quantidade correspondente a três vezes a massa do substrato, e colocadas em germinador, a $30^{\circ} \mathrm{C}$. As contagens foram feitas no $10^{\circ}$ e $18^{\circ}$ dias após a instalação e os 
resultados foram expressos em percentagem de plântulas normais (Lopes \& Pereira 2005); 3) Primeira contagem de germinação: conduzida em conjunto com o teste de germinação, consistiu no registro do número de plântulas normais obtidas no $10^{\circ}$ dia após o início do teste de germinação; 4) Comprimento de plântulas: no $18^{\circ}$ dia após a instalação do teste de quatro repetições de 20 plântulas, foram medidos, em centímetros, com o auxílio de uma régua graduada, o comprimento de plântulas (raiz + parte áerea); 5) Teste de emergência: quatro parcelas de 50 sementes foram semeadas, a $5 \mathrm{~mm}$ de profundidade, em caixas plásticas com areia, como substrato. As caixas foram mantidas no laboratório até a estabilização da emergência, quando foi determinada a percentagem de plântulas emergidas; 6) Índice de velocidade de emergência: realizado juntamente com o teste de emergência, nele contou-se, diariamente, o número de plântulas normais, até o $18^{\circ}$ dia após a semeadura, conforme metodologia proposta por Maguire (1962); 7) Envelhecimento acelerado: foram estudadas três variações na temperatura $\left(41^{\circ} \mathrm{C}, 42^{\circ} \mathrm{C}\right.$ e $\left.45^{\circ} \mathrm{C}\right) \mathrm{e}$ quatro períodos de envelhecimento (24, 48, 72 e 96 horas), em delineamento inteiramente casualizado, em esquema fatorial, com quatro repetições, de 50 sementes fisicamente puras. As sementes foram previamente pesadas e colocadas para envelhecer em caixas gerbox, com tela contendo $40 \mathrm{~mL}$ de água destilada, e mantidas em câmaras tipo B.O.D, ajustadas às temperaturas e períodos estudados. Após cada período de envelhecimento, a germinação de cada lote, em cada um dos tratamentos, foi avaliada, seguindo-se a metodologia descrita anteriormente para o teste de germinação, com contagem aos 10 dias.

Os dados foram submetidos a análise de variância e as médias dos tratamentos comparadas pelo teste Tukey, a $5 \%$ de probabilidade, efetuadas pelo programa SAS.

\section{RESULTADOS E DISCUSSÃO}

Em relação à caracterização inicial da qualidade fisiológica das sementes de cubiu (Tabela 1), observa-se que não houve diferença estatística significativa na germinação entre os quatro lotes. Entretanto, na primeira contagem de germinação, pôde-se observar o melhor desempenho dos lotes 3 e 4, que diferiram dos demais lotes, mostrando-se mais vigorosos que os lotes 1 e 2. Para as características de vigor relacionadas à emergência e ao índice de velocidade de germinação, os lotes 1 e 2 também apresentaram qualidade inferior aos demais. A emergência de plântuas também estratificou os lotes em dois níveis de vigor, sendo os lotes 3 e 4 aqueles que apresentaram maior percentagem de plântulas, ao final do ensaio. Portanto, de acordo com a caracterização da qualidade fisiológica dos lotes de sementes de cubiu, constatou-se que, na maioria dos testes empregados, houve semelhança quanto ao alto vigor dos lotes 3 e 4 e menor vigor dos lotes 1 e 2 . Cabe ressaltar que o teste de germinação não conseguiu ordenar os lotes, quanto ao nível de vigor, já que a germinação é a última característica fisiológica afetada pelo processo de deterioração das sementes, não sendo eficiente para detectar diferenças entre lotes de sementes (Marcos Filho 2005).

Pelo teste de envelhecimento acelerado a $41^{\circ} \mathrm{C}$ (Tabela 2), verifica-se, em todos os períodos estudados, melhor desempenho do lote 3 . Nos períodos de 72 e 96 horas, foi evidenciado o maior grau de germinação para as sementes dos lotes 3 e 4 , respectivamente, ficando os demais em posição inferior. Estes resultados são semelhantes aos encontrados nos testes de caracterização dos lotes de cubiu, principalmente o de primeira contagem de germinação e o de emergência de plântulas. Observa-se, ainda, que, após 96 horas de envelhecimento, não houve

Tabela 1. Caracterização da qualidade fisiológica inicial de sementes de cubiu (Solanum sessiliflorum Dunal), pelo teor de água (U), germinação (G), primeira contagem de germinação (PCG), comprimento de plântula (CP), emergência (E) e índice de velocidade de germinação (IVG) (Viçosa, MG, 2009).

\begin{tabular}{ccccccc}
\hline Lotes & $\mathrm{U}(\%)$ & $\mathrm{G}(\%)$ & $\mathrm{PC}(\%)$ & $\mathrm{CP}(\mathrm{cm})$ & $\mathrm{E}(\%)$ & $\mathrm{IVE}$ \\
\hline 1 & $8,0 \mathrm{a}^{*}$ & $96 \mathrm{a}$ & $38 \mathrm{~b}$ & $0,81 \mathrm{~b}$ & $30 \mathrm{~b}$ & $4,27 \mathrm{~b}$ \\
2 & $7,6 \mathrm{a}$ & $92 \mathrm{a}$ & $42 \mathrm{~b}$ & $1,23 \mathrm{ab}$ & $26 \mathrm{~b}$ & $3,12 \mathrm{~b}$ \\
3 & $7,9 \mathrm{a}$ & $98 \mathrm{a}$ & $64 \mathrm{a}$ & $2,21 \mathrm{a}$ & $42 \mathrm{a}$ & $6,83 \mathrm{a}$ \\
4 & $8,2 \mathrm{a}$ & $100 \mathrm{a}$ & $66 \mathrm{a}$ & $1,97 \mathrm{ab}$ & $46 \mathrm{a}$ & $5,79 \mathrm{ab}$ \\
\hline $\mathrm{CV}(\%)$ & 2,74 & 6,83 & 5,38 & 4,61 & 9,42 & 5,43 \\
\hline
\end{tabular}

* Médias seguidas pela mesma letra, na coluna, não diferem pelo Teste Tukey, a 5\% de probabilidade. 
redução drástica na percentagem de germinação. Portanto, foi possível associar alguns resultados do teste de envelhecimento acelerado a $41^{\circ} \mathrm{C}$ a resultados semelhantes, em outros testes de vigor (Tabela 1). Em sementes de melão, Torres \& Marcos Filho (2003) verificaram que o envelhecimento acelerado a $41^{\circ} \mathrm{C}$ foi sensível para se detectar diferenças na qualidade fisiológica dos lotes.

Nos testes de envelhecimento acelerado conduzidos a $42^{\circ} \mathrm{C}$ e $45^{\circ} \mathrm{C}$ (Tabela 2), verifica-se que não houve estratificação homogênea dos lotes e nem paridade com os outros testes de vigor aos quais os lotes foram submetidos. Nota-se que, nestas temperaturas, os lotes foram separados em três categorias, o que não aconteceu na temperatura de $41^{\circ} \mathrm{C}$ e nos testes de caracterização do vigor das sementes. Segundo Marcos Filho (1999), a identificação do potencial fisiológico dos lotes de médio vigor é, muitas vezes, mais difícil, pois estes lotes podem apresentar variações no seu comportamento, ora se igualando aos lotes de maior

Tabela 2. Percentagem de germinação de sementes de cubiu (Solanum sessiliflorum Dunal), obtidas de quatro lotes, por ocasião da exposição ao envelhecimento acelerado por quatro períodos $(24,48,72$ e 96 horas $)$ e três temperaturas $\left(41^{\circ} \mathrm{C}, 42^{\circ} \mathrm{C}\right.$ e $45^{\circ} \mathrm{C}$ ) (Viçosa, MG, 2009).

\begin{tabular}{|c|c|c|c|c|}
\hline \multirow{2}{*}{ Lote } & \multicolumn{4}{|c|}{$41^{\circ} \mathrm{C}$} \\
\hline & $24 \mathrm{~h}$ & $48 \mathrm{~h}$ & $72 \mathrm{~h}$ & $96 \mathrm{~h}$ \\
\hline & \multicolumn{4}{|c|}{$\%$} \\
\hline $\begin{array}{l}1 \\
2\end{array}$ & $\begin{array}{l}45 b^{*} \\
28 \mathrm{c}\end{array}$ & $\begin{array}{l}41 \mathrm{~b} \\
55 \mathrm{ab}\end{array}$ & $\begin{array}{l}28 \mathrm{~b} \\
12 \mathrm{c}\end{array}$ & $\begin{array}{l}32 \mathrm{~b} \\
36 \mathrm{~b}\end{array}$ \\
\hline 3 & $72 \mathrm{a}$ & $70 \mathrm{a}$ & $73 \mathrm{a}$ & $78 \mathrm{a}$ \\
\hline 4 & $50 \mathrm{~b}$ & $46 \mathrm{~b}$ & $69 \mathrm{a}$ & $76 \mathrm{a}$ \\
\hline $\mathrm{CV}(\%)$ & 7,3 & 8,2 & 5,8 & 11,5 \\
\hline \multirow{2}{*}{ Lote } & \multicolumn{4}{|c|}{$42^{\circ} \mathrm{C}$} \\
\hline & $24 \mathrm{~h}$ & $48 \mathrm{~h}$ & $72 \mathrm{~h}$ & $96 \mathrm{~h}$ \\
\hline & \multicolumn{4}{|c|}{$-\%$} \\
\hline 1 & $23 \mathrm{~b}$ & $55 \mathrm{~b}$ & $53 \mathrm{~b}$ & $50 \mathrm{bc}$ \\
\hline 2 & $28 \mathrm{~b}$ & $57 \mathrm{ab}$ & $08 \mathrm{c}$ & $55 \mathrm{~b}$ \\
\hline 3 & $49 a b$ & $71 \mathrm{a}$ & $72 a$ & $68 \mathrm{a}$ \\
\hline 4 & $60 \mathrm{a}$ & $54 \mathrm{~b}$ & $52 \mathrm{~b}$ & $42 \mathrm{c}$ \\
\hline $\mathrm{CV}(\%)$ & 9,1 & 5,4 & 6,9 & 4,7 \\
\hline \multirow{2}{*}{ Lote } & \multicolumn{4}{|c|}{$45^{\circ} \mathrm{C}$} \\
\hline & $24 \mathrm{~h}$ & $48 \mathrm{~h}$ & $72 \mathrm{~h}$ & $96 \mathrm{~h}$ \\
\hline & \multicolumn{4}{|c|}{$-\%$} \\
\hline 1 & $10 \mathrm{c}$ & $52 \mathrm{~b}$ & $21 \mathrm{bc}$ & $20 \mathrm{bc}$ \\
\hline 2 & $07 \mathrm{c}$ & $50 \mathrm{~b}$ & $09 \mathrm{c}$ & $07 \mathrm{c}$ \\
\hline 3 & $63 \mathrm{a}$ & $71 \mathrm{a}$ & $62 \mathrm{a}$ & $67 \mathrm{a}$ \\
\hline 4 & $37 \mathrm{~b}$ & $52 \mathrm{~b}$ & $38 \mathrm{~b}$ & $32 \mathrm{~b}$ \\
\hline $\mathrm{CV}(\%)$ & 8,4 & 7,9 & 6,3 & 8,8 \\
\hline
\end{tabular}

vigor, ora aos de menor vigor, dependendo do teste de vigor empregado e do ajuste da metodologia. Como não houve homogeneidade desta divisão em três níveis de vigor, em todos os períodos de embebição, pode ter ocorrido deterioração de forma exagerada, quando os lotes foram submetidos às condições de estresse.

Também Bhering et al. (2003), trabalhando com sementes de melancia, e Carpi (2005), com sementes de rabanete, não obtiveram separação eficiente de lotes, quanto ao vigor, utilizando temperaturas mais altas, como $42^{\circ} \mathrm{C}$ e $45^{\circ} \mathrm{C}$. Da mesma maneira, Rodo et al. (2000), com sementes de cenoura; Torres \& Marcos Filho (2003), com melão; e Rodo \& Marcos Filho (2003), com cebola, verificaram que o uso de temperaturas acima de $42^{\circ} \mathrm{C}$ proporcionou resultados menos uniformes, afetando a eficiência do teste. Por sua vez, Panobianco \& Marcos Filho (2001) e Dutra (2004) constataram que, em temperatura de $41^{\circ} \mathrm{C}$, houve uma classificação dos lotes de tomate e abóbora, respectivamente, em níveis de vigor, indicando como mais adequada a combinação de $41^{\circ} \mathrm{C}$, por 72 horas.

Os dados referentes ao teor de água das sementes, antes e após o envelhecimento acelerado, encontram-se na Tabela 3. Verificou-se que, comparado o teor de água inicial das sementes dos 4 lotes (Tabela 1) com o de 48 horas de envelhecimento, as sementes absorveram água, atingindo valores entre $13,7 \%$ e $35,4 \%$, a $41^{\circ} \mathrm{C}$, e entre $12,5 \%$ e $35,8 \%$, a $42^{\circ} \mathrm{C}$, com elevação destes valores, com o aumento da temperatura para $45^{\circ} \mathrm{C}$ (entre $16,6 \%$ e $37,2 \%$ ). $\mathrm{O}$ aumento do período de envelhecimento proporcionou aumento no teor de água das sementes.

O grau de umidade das sementes, após o envelhecimento acelerado, é um dos principais indicadores da uniformidade das condições de envelhecimento, pois variações de três a quatro pontos percentuais entre amostras são toleráveis (Marcos Filho 1999). Verifica-se que, na maioria das condições estudadas neste trabalho, a variação entre o teor de água das sementes dos seis lotes não permaneceu, em geral, dentro do limite tolerável, que é de 2 a 4 pontos percentuais.

De acordo com Carvalho \& Nakagawa (2000), incrementos nos teores de água favorecem a elevação da temperatura da semente, em decorrência dos processos respiratórios e da maior atividade de microorganismos. $\mathrm{O}$ aumento no tempo de exposição ao envelhecimento acelerado pode ter proporcionado 
Tabela 3. Teor de água (\%) de sementes de cubiu (Solanum sessiliflorum Dunal), obtido de quatro lotes, por ocasião da exposição ao envelhecimento acelerado, por quatro períodos $(24,48,72$ e 96 horas) e três temperaturas $\left(41^{\circ} \mathrm{C}, 42^{\circ} \mathrm{C}\right.$ e $\left.45^{\circ} \mathrm{C}\right)$ (Viçosa, $\mathrm{MG}$, 2009).

\begin{tabular}{|c|c|c|c|c|}
\hline \multirow{3}{*}{ Lote } & \multicolumn{4}{|c|}{$41^{\circ} \mathrm{C}$} \\
\hline & $24 \mathrm{~h}$ & $48 \mathrm{~h}$ & $72 \mathrm{~h}$ & $96 \mathrm{~h}$ \\
\hline & \multicolumn{4}{|c|}{$\%$} \\
\hline 1 & 13,7 & 18,7 & 29,3 & 34,7 \\
\hline 2 & 16,4 & 19,2 & 28,7 & 33,4 \\
\hline 3 & 15,7 & 20,8 & 32,4 & 35,2 \\
\hline 4 & 15,3 & 23,0 & 30,8 & 35,4 \\
\hline \multirow[t]{2}{*}{$\mathrm{CV}(\%)$} & 4,6 & 7,9 & 8,3 & 7,5 \\
\hline & \multicolumn{4}{|c|}{$42^{\circ} \mathrm{C}$} \\
\hline \multirow{2}{*}{ Lote } & $24 \mathrm{~h}$ & $48 \mathrm{~h}$ & $72 \mathrm{~h}$ & $96 \mathrm{~h}$ \\
\hline & \multicolumn{4}{|c|}{$\%$} \\
\hline 1 & 12,8 & 23,7 & 30,1 & 35,4 \\
\hline 2 & 15,7 & 27,2 & 32,2 & 36,2 \\
\hline 3 & 16,8 & 26,5 & 33,7 & 37,1 \\
\hline 4 & 16,7 & 24,0 & 34,6 & 35,8 \\
\hline \multirow[t]{2}{*}{$\mathrm{CV}(\%)$} & 8,9 & 8,3 & 6,9 & 7,4 \\
\hline & \multicolumn{4}{|c|}{$45^{\circ} \mathrm{C}$} \\
\hline \multirow{2}{*}{ Lote } & $24 \mathrm{~h}$ & $48 \mathrm{~h}$ & $72 \mathrm{~h}$ & $96 \mathrm{~h}$ \\
\hline & \multicolumn{4}{|c|}{$\%$} \\
\hline 1 & 16,8 & 32,3 & 36,7 & 36,7 \\
\hline 2 & 16,5 & 33,9 & 35,2 & 37,2 \\
\hline 3 & 15,8 & 33,5 & 33,4 & 36,2 \\
\hline 4 & 16,4 & 35,6 & 35,3 & 35,3 \\
\hline $\mathrm{CV}(\%)$ & 7,4 & 9,1 & 11,6 & 10,5 \\
\hline
\end{tabular}

maior incremento no teor de umidade, nas sementes condicionadas. Este fato, aliado à temperatura elevada $\left(45^{\circ} \mathrm{C}\right)$ imposta pelo teste de envelhecimento, resultou em um processo de deterioração mais acelerado destas sementes, do que das sementes submetidas a temperaturas menores.

\section{CONCLUSÕES}

1. O teste de envelhecimento acelerado é eficiente na separação de lotes, em diferentes níveis de vigor, empregando-se a temperatura de $41^{\circ} \mathrm{C}$, por 72 ou 96 horas.

2. Sementes de cubiu tiveram sua viabilidade reduzida, quando submetidas ao teste de envelhecimento acelerado, devido ao aumento considerável da taxa de deterioração das sementes, através da exposição a níveis muito adversos de temperatura e umidade relativa.

\section{REFERENNCIAS}

ASSOCIATION OF OFFICIAL SEED ANALYSTS (AOSA). Seed vigor testing handbook. East Lansing: AOSA, 1983.

BHERING, M. C. et al. Avaliação do vigor de sementes de melancia (Citrullus lanatus Scherad) pelo teste de envelhecimento acelerado. Revista Brasileira de Sementes, Londrina, v. 25, n. 2, p. 1-6, 2003.

BHERING, M. C. et al. Métodos para avaliação do vigor de sementes de pepino. Revista Brasileira de Sementes, Londrina, v. 22, n. 2, p. 171-175, 2000.

BRASIL. Ministério da Agricultura e Reforma Agrária. Regras para análise de sementes. Brasília: SNDA/DNDV/ CLAV, 2009.

CARPI, V. A. F. Avaliação do potencial fisiológico de sementes de rabanete (Raphanus sativus L.). 2005. $77 \mathrm{f}$. Tese (Doutorado em Agronomia)-Escola Superior de Agricultura "Luiz de Queiroz", Universidade de São Paulo, Piracicaba, 2005.

CARVALHO, N. M. de; NAKAGAWA, J. Sementes: ciência, tecnologia e produção. 4. ed. Jaboticabal: Funep, 2000 .

DELOUCHE, J. C.; BASKIN, C. C. Accelerated aging techniques for predicting the relative storability of seed lots. Seed Science and Technology, Zürich, v. 1, n. 3, p. 427-452, 1973.

DIAS, D. C. F. S.; MARCOS FILHO, J. Testes de condutividade elétrica para avaliação do vigor de sementes de soja (Glycine max (L.) Merrill). Scientia Agricola, Piracicaba, v. 53, n. 1, p. 31-42, 1996.

DUTRA, A. S. Avaliação do potencial fisiológico de sementes de abóbora e abobrinha. 2004. 76 f. Tese (Doutorado em Agronomia)-Faculdade de Ciências Agrárias e Veterinárias, Universidade Estadual Paulista, Jaboticabal, 2004.

FRANZIN, S. M. et al. Avaliação do vigor de sementes de alface nuas e peletizadas. Revista Brasileira de Sementes, Londrina, v. 26, n. 2, p. 114-118, 2004.

LOPES, J. C.; PEREIRA, J. C. Germinação de sementes de cubiu em diferentes substratos e temperaturas. Revista Brasileira de Sementes, Londrina, v. 27, n. 2, p. 146-150, 2005.

MAGUIRE, J. D. Speed of germination and in selection and evaluation for seeding emergence and vigor. Crop Science, Madison, v. 2, n. 2, p. 176-177, 1962.

MARCOS FILHO, J. Fisiologia de sementes de plantas cultivadas. Piracicaba: Fealq, 2005. 
MARCOS FILHO, J. Teste de envelhecimento acelerado. In: KRZYZANOWSKI, F. C.; VIEIRA, R. D.; FRANÇA NETO, J. B. (Eds.). Vigor de sementes: conceitos e testes. Londrina: Abrates, 1999. p. 1-24.

PANOBIANCO, M.; MARCOS FILHO, J. Comparação entre métodos para avaliação da qualidade fisiológica de sementes de pimentão. Revista Brasileira de Sementes, Londrina, v. 20, n. 2, p. 306-310, 1998.

PANOBIANCO, M.; MARCOS FILHO, J. Envelhecimento acelerado e deterioração controlada em sementes de tomate. Scientia Agricola, Piracicaba, v. 58, n. 3, p. 525531, 2001.

PIANA, Z.; TILLMANN, M. A. A.; MINAMI, K. Avaliação da qualidade fisiológica de sementes de cebola e sua relação com a produção de mudas vigorosas. Revista Brasileira de Sementes, Londrina, v. 20, n. 2, p. 301-305, 1995.

RAMOS, N. P. et al. Envelhecimento acelerado em sementes de rúcula (Eruca sativa L.). Revista Brasileira de Sementes, Londrina, v. 26, n. 1, p. 98-103, 2004.

RIBEIRO, F. C. Comparação entre os sistemas de envelhecimento artificial de sementes através de soluções salinas e o tradicional em cenoura (Daucus carota L.), alface (Lactuca sativa L.) e brócolos (Brassica oleracea variedade etálica Plenk.). 2000. 56 f. Dissertação (Mestrado em Agronomia)-Faculdade de Ciências Agrárias e Veterinárias, Universidade Estadual Paulista, Jaboticabal, 2000.

RODO, A. B.; MARCOS FILHO, J. Onion seed vigor in relation to plant growth and yield. Horticultura Brasileira, Brasília, DF, v. 21, n. 2, p. 220-226, 2003.

RODO, A. B.; PANOBIANCO, M.; MARCOS FILHO, J. Metodologia alternativa do teste de envelhecimento acelerado para sementes de cenoura. Scientia Agricola, Piracicaba, v. 57, n. 2, p. 289-292, 2000.
RODO, A. B.; TILLMANN, M. A. A.; VILlELA, F. A. Testes de vigor na avaliação da qualidade fisiológica de sementes de tomate. Revista Brasileira de Sementes, Londrina, v. 20, n. 1, p. 23-38, 1998.

SILVA FILHO, D. F. Discriminação de etnovariedade de cubiu (Solanum sessiliflorum Dunal, Solanaceae) da Amazônia, com base em suas características morfológicas e químicas. 2002. $117 \mathrm{f}$. Tese (Doutorado em Ciências Biológicas - Botânica)-Instituto Nacional de Pesquisas da Amazônia, Universidade Federal do Amazonas, Manaus, 2002.

SILVA FILHO, D. F. Manual técnico cocona (Solanum sessiliflorum Dunal): cultivo y utilización. Caracas: Secretaria Pro-tempore, 1998.

SILVA FILHO, D. F. Orientações técnicas para o cultivo do cubiu (Solanum sessiliflorum Dunal) na Amazônia. Manaus: INPA, 1996.

SILVA FILHO, D. F. et al. Análise multivariada da divergência genética em 29 populações de cubiu (Solanum sessiliflorum Dunal) avaliada na zona da mata do Estado de Pernambuco. Acta Amazonica, Manaus, v. 25, n. 3/4, p. 171-180, 1995.

SPINOLA, M. C. M. et al. Comparação entre métodos para avaliação do vigor de sementes de cenoura. Revista Brasileira de Sementes, Londrina, v. 20, n. 2, p. 301-305, 1998.

TORRES, S. B.; CARVALho, I. M. S. Teste de envelhecimento acelerado em sementes de quiabo (Albelmoschus esculentus (L.) Moench). Revista Brasileira de Sementes, Londrina, v. 20, n. 1, p. 209-211, 1998.

TORRES, S. B.; MARCOS FILHO, J. Accelerated aging of melon seeds. Scientia Agricola, Piracicaba, v. 60, n. 1, p. 77-82, 2003. 